\title{
BMJ Open Quality Improving time efficiency gathering equipment in the treatment room
}

\author{
Yesim Karapinar, Ali Habib, Hannah Sawyerr
}

To cite: Karapinar Y, Habib A, Sawyerr H. Improving time efficiency gathering equipment in the treatment room.BMJ Open Quality 2017;6:e000010. doi:10.1136/ bmjoq-2017-000010

Received 18 January 2017 Revised 26 August 2017 Accepted 24 September 2017

CrossMark

Department of Medicine, Northampton General Hospital NHS Trust, Northampton, UK

Correspondence to Dr Yesim Karapinar; y.karapinar@nhs.net

\section{ABSTRACT}

Each junior doctor spends on average 29 hours a year just accessing treatment rooms and approximately 4 working days per year collecting equipment. We identified areas where time efficiency could be improved: accessing treatment room door codes, standardising access to equipment in treatment rooms throughout the hospital, implementing the 'procedure-specific' tray (one tray per procedure which includes all needed equipment in one place) and indexing equipment. Our aim was to reduce the time taken to collect equipment, promote best practice and aid timely medical intervention. We collected data from 24 junior doctors with a Likert scale questionnaire, which confirmed the problem. We then designed an experiment where we timed healthcare professionals accessing treatment rooms with no prior knowledge of the codes and then with codes provided securely on our hospital-issued iPad. We project a time saving of 703 hours (88 working days) at Northampton General Hospital (NGH) over 1 year. We then implemented our prototype 'procedure-specific trays' for common procedures (cannulation, lumbar puncture, catheter). We calculated how much time was saved when collecting equipment using our indexed 'procedure-specific' tray method compared with current practice. Based on our piloted trays, we project to save a total of 802 hours ( 100 working days) at NGH over 1 year. To finalise our project, we trialled our custom design trays based on our prototype for cannulation and demonstrated a time saving of $97 \%$ relative improvement: from $225 \mathrm{~s}$ to $7 \mathrm{~s}$. According to these results, once full roll of 'procedurespecific tray' is achieved, the trust will save projected $£ 30100$ per year (based on average junior doctor salary of $£ 20$ per hour).

\section{PROBLEM}

This quality improvement (QI) project was undertaken at Northampton General Hospital (NGH). This is a 765-acute-bed district general hospital with 92 junior doctors (Foundation Year 1 (FY1), Core Trainee 2 (CT2)) level. NGH serves a city population of 350000 and a wider population of 880000 .

We calculated that each junior doctor spends on average 29 hours a year just accessing treatment rooms and 4 working days ( 8 hour days) per year collecting equipment. This was calculated from a survey of junior doctors over an 8-hour shift.

The QI project was borne out of personal experiences. The project which was named initially 'The Treatment Room' was initiated by merging of two projects. The first followed an on-call shift where an FY1 was required to take blood tests on different wards and was not able to locate readily the equipment needed in the treatment rooms. The second initiated following a periarrest where the FY1 was asked to get additional equipment from a treatment room; due to the door codes not being readily available, vital time was wasted delaying access to the treatment room. These were the initial catalyst for this QI project.

As junior doctors, we found it difficult to access treatment rooms and struggle to locate specific equipment to carry out common procedures.

We identified a lack of standardisation throughout the treatment rooms, which drove us to quantify the extent of the problem.

Questionnaires (table 1) were issued to 26 junior doctors, and $24(92 \%)$ responses were received.

Through this project, we aimed to make treatment room access codes readily available to all junior doctors. By standardising the treatment room, we wanted to reduce time taken to collect equipment by $25 \%$. Overall, our aim was to improve time efficiency and staff well-being in order to improve patient care and safety by providing timely medical intervention.

Overall general feedback included improving stocking and organisation; better labelling of equipment; organising equipment by procedure, as well as a checklist for procedures.

Figure 1 is a driver diagram for establishing the reason why change to the treatment room equipment organisation is required.

We were driven to tackle this problem (figure 1: driver diagram) by the potential gain of reducing stress, wastage of equipment and working in a tidy organised space in order to provide timely medical intervention.

Our SMART aim was to reduce time taken to collect equipment for cannulation, venepuncture, lumbar puncture and urinary catheter by $25 \%$ by August 2016 on acute 
Table 1 Junior doctor Likert scale questionnaire: quantifying Issues regarding time efficiency gathering equipment in the treatment room

\begin{tabular}{|c|c|c|c|c|c|}
\hline & Strongly disagree & Disagree & Undecided & Agree & $\begin{array}{l}\text { Strongly } \\
\text { agree }\end{array}$ \\
\hline It is easy to access all treatment rooms while on call & 5 & 13 & 3 & 3 & 0 \\
\hline Equipment is in the same place in each treatment room & 14 & 9 & 1 & 0 & 0 \\
\hline $\begin{array}{l}\text { I know all the equipment I need to gather for all common } \\
\text { procedures }\end{array}$ & 4 & 6 & 5 & 9 & 0 \\
\hline Current treatment rooms require no improvement & 11 & 12 & 1 & 0 & 0 \\
\hline
\end{tabular}

admissions ward. We decided on $25 \%$ time reduction as we felt this was an achievable aim.

\section{BACKGROUND}

Likert scale questionnaires issued to junior doctors confirmed the difficulty assessing treatment rooms and gathering equipment to be a unifying problem. Literature review identified this to be a common problem across many hospital trusts within the UK. ${ }^{1}$

Previous attempts at resolving this issue included making available equipment for cannulation and venepuncture on a single trolley. ${ }^{2}$ Another example took this concept further by increasing the number of procedures (arterial blood gas and blood culture) catered for in one trolley. ${ }^{3}$

Rearranging and labelling equipment in order to make equipment more accessible had been attempted on a children's ward. The project demonstrated that improvement in efficiency can be made with little resources. ${ }^{4}$

There has been a previous attempt in resolving this problem in NGH. A clear system of labelling equipment was introduced numbering the trays that contained the components for venepuncture. The aim was to improve patient safety by providing timely medical intervention. The project was unsuccessful at rolling out due to time constraints. $^{5}$

\section{BASELINE MEASUREMENT}

To assess the extent of the problem we issued 26 Likert scale questionnaires to junior doctors and received 24 completely filled responses. The questionnaire was designed to collect both quantitative and qualitative data.

Quantitative data showed that 18 (75\%) respondents either strongly disagreed or disagreed to the treatment rooms being easily accessible during on-call shifts with a further $3(12.5 \%)$ being undecided. Twenty-three $(96 \%)$ of respondents felt equipment was not in the same place in each treatment room. Ten $(41 \%)$ of those surveyed strongly disagreed or disagreed that they felt confident that they were aware of all the components required to carry out common medical procedures. Overall a significant number, $23(96 \%)$ felt that the current treatment rooms needed improvement to address these shortcomings.

General comments from respondents included a need to improve stocking and organisation of the current treatment room. Many felt that gathering equipment in the current treatment room was inefficient and caused unnecessary time delay, adversely affecting timely medical intervention. Furthermore, suggestions included better labelling and incorporating a colour-coded system. One respondent asked for a checklist for common procedures to ensure all equipment was gathered in one attempt.

For baseline measurements, we conducted timed trials on healthcare professionals who routinely use medical equipment. This included junior doctors, medical students, nurses and physician associates. The initial trial timed how long it took for a participant to enter the treatment room without prior knowledge of the treatment rooms code. Participants started at a predetermined point, on the same ward, and were tasked to access the same treatment room to reduce variables. Sixteen participated with mean time taken of $119 \mathrm{~s}$ (range 82-167s).

The second baseline trial involved timing participants assembling a cannulation tray in the current treatment room layout. Again, starting point, locations of equipment and location of treatment room were kept constant. Nine participants had a mean time of $150 \mathrm{~s}$ (range 88-225s).

We also conducted baseline measurements for collecting equipment for urinary catheterisation. Seven participants gathered the equipment in a mean time of 93s (range 83-114s).

Seven participants attempted to gather equipment for lumbar puncture of whom five failed to gather all necessary equipment. Only two participants were successful in gathering all required equipment with the mean time being 335 s (range 293-378s).

\section{DESIGN}

We wanted to make a cost-effective and relatively simple solution for our QI project. We needed a solution that was reproducible on a hospital-wide level. 


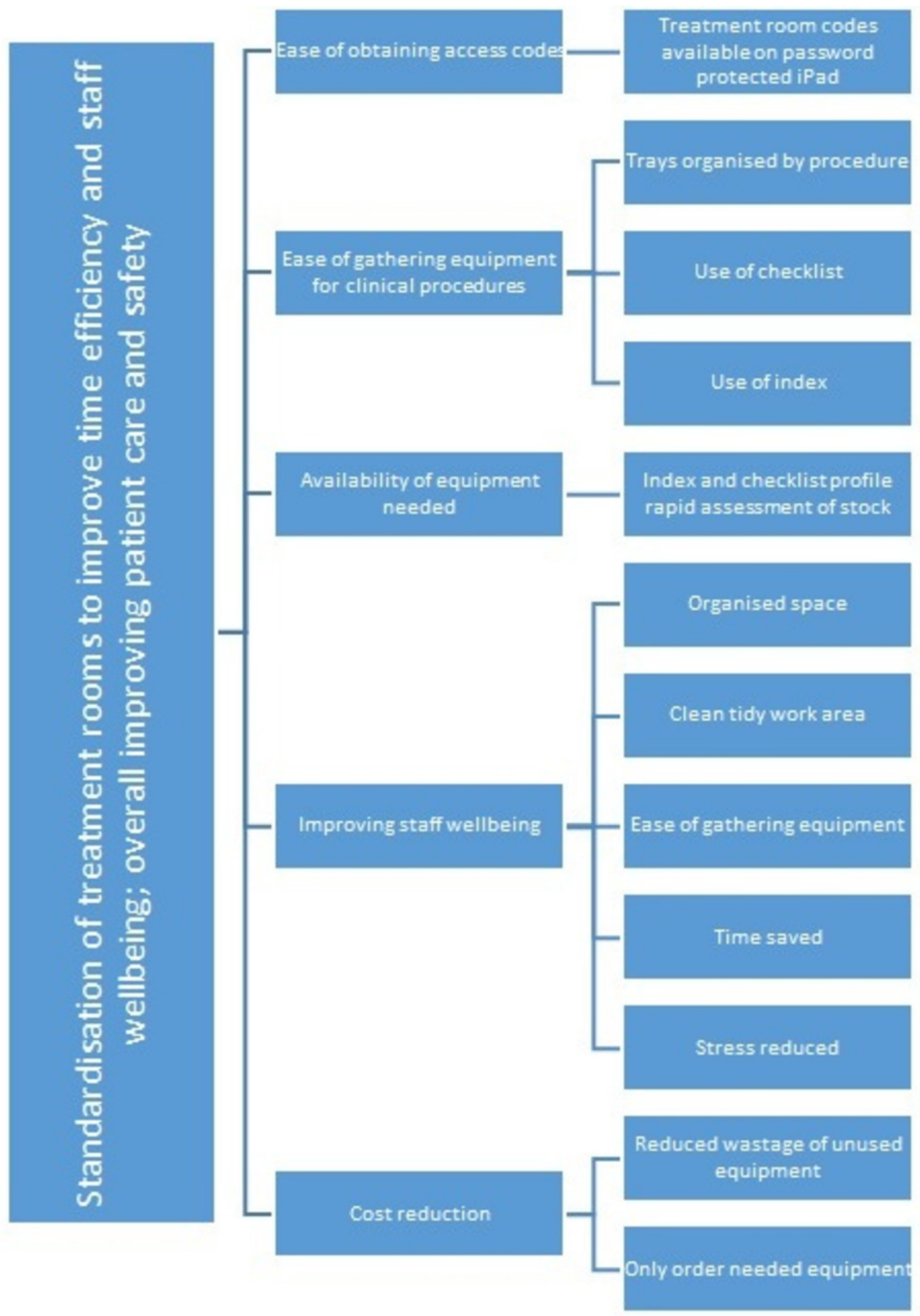

Figure 1 Junior doctor questionnaire: quantifying issues with the treatment room.

We decided that the simplest and most cost-effective solution to make treatment room codes accessible to junior doctors in a confidential and secure manner was to make them available on the trust-issued iPad that each junior doctor was given.

As for the treatment rooms, we were inspired by our previous experiences in other hospitals and looked at off-the-shelf commercial solutions.

We came up with the idea of the 'procedure-specific' tray. In this concept, all relevant equipment for a given procedure, for example, cannulation was stocked in a single tray, eliminating the need to locate the various individual components (an often cumbersome and time-consuming process).

We used tray dividers to compartmentalise our trays and placed individual equipment in the order they were to be used in the trays. We also added a 'crib sheet'; this listed all the equipment required for the procedure.

For our prototype, we used the existing trays and selfmade cardboard box tray dividers. We then ran a PlanDo-Study-Act (PDSA) cycle and compared our new tray prototype with the existing treatment room trays. We 
showed a significant reduction in time taken to gather equipment thus validating our concept.

We engaged with various stakeholders in the hospital. We sought permission from the ward sister to implement the trays on the ward.

We involved the infection control lead in making sure our trays were in accordance with hospital infection control guidelines, which led to prototype 2. We laminated the dividers and the 'crib sheets', ensuring it was a wipeable surface. We presented our project to the hospital's executive board and succeeded in gathering support and funding for the project. We involved estates/procurement in evaluating how our project can be implemented within existing hospital architecture.

We involved industry for the final iteration of our tray. We have customised them with tray dividers and labels that are sturdy and conform to infection control guidelines.

In conjuncture with the lead for patient safety at NGH, we have come up with a 'scale-up and roll-out strategy.'

Initially, we will implement these trays on the acute medical admissions unit where we first gathered our results. After collecting feedback, we will run another PDSA cycle and make implementations based on our feedback. We then plan to roll out on the surgical wards as the treatment rooms are architecturally identical. Finally, we will standardise all remaining wards in the hospital with the procedure-specific trays.

This scale-up and roll-out strategy makes sure we can make a permanent and sustainable change.

\section{STRATEGY}

We based our project on the PDSA model (figure 2) for QI.

Initially, we gathered baseline data, simulating how long it takes to enter the current treatment rooms at NGH.

In order to control variation when measuring baseline measurement, we ensured we timed participants walking from a predetermined point and stopped timing once they successfully entered the treatment room.

Our first incremental improvement was to make available the code on a hospital iPad. We retimed the same participant and found that having the codes readily available saved an average of $31 \mathrm{~s}$ for each participant.

Our second PDSA cycle consisted of timing participants gathering cannulation equipment in the current treatment room layout. We then retimed the same participants with the procedure-specific tray for cannula. The procedure-specific tray saved an average of $121 \mathrm{~s}$.

Further PDSA cycles included adding a crib sheet. This was attached to the tray and listed all the equipment required for the procedure. We trialled this with gathering equipment for lumbar puncture. Without the crib sheet, many of the participants did not know what equipment was required and many abandoned the task.

With the crib sheet, $100 \%$ of all participants gathered the required equipment. We found a $71 \%$ average time reduction in time taken to gather lumbar puncture equipment. After implementing our 'procedure-specific tray', we were informed by the infection control team about the

\section{Improve efficiency of treatment rooms}

\section{Decreased time/effort in gathering equipment}

\section{Improve staff and Patient outcomes}

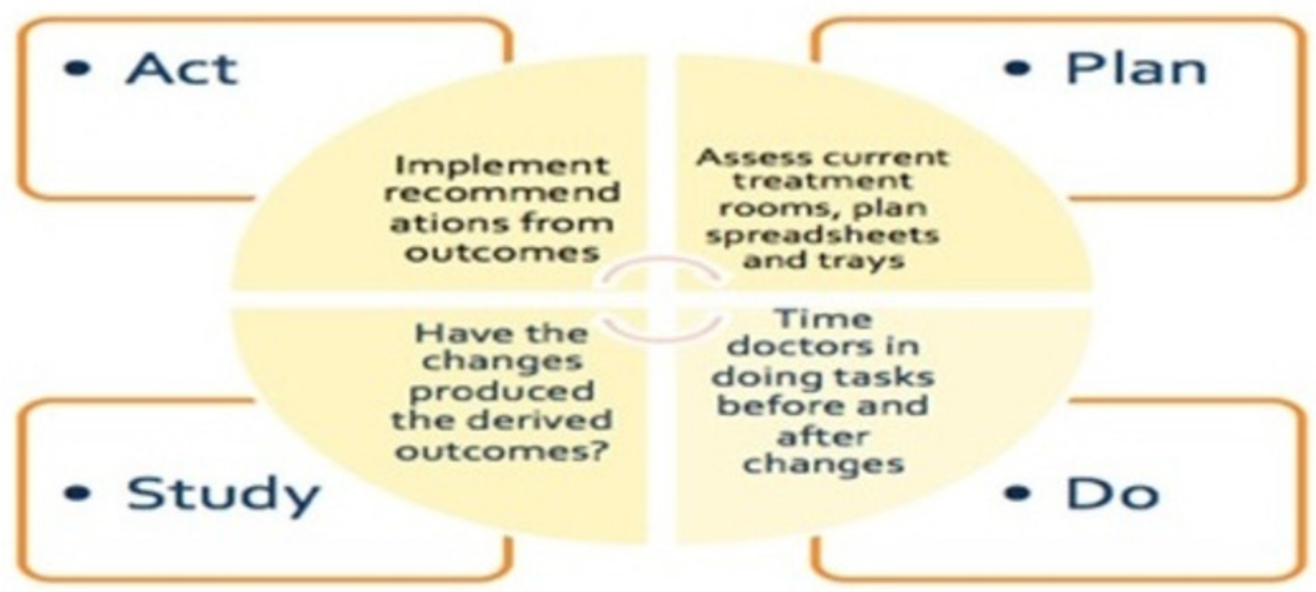

Figure 2 Plan-Do-Study-Act cycle. 
health and safety requirements for storing equipment on the ward. To overcome this, we bought customised plastic tray dividers (to replace the cardboard dividers) and trailed these. Thirteen participants were timed gathering equipment for the procedure cannulation, and similar results were obtained.

\section{RESULTS}

PDSA cycle 1 was making door codes available on our trust-issued iPads and timing participants accessing the treatment room. The controls included a fixed starting point and the treatment room that was accessed. Sixteen participated gained access to the treatment room in a mean time of $88 \mathrm{~s}$ (range 61-140s). After intervention, we showed a mean reduction of $31 \mathrm{~s}$ (range).

PDSA cycle 2 involved introducing 'procedure-specific trays' within the treatment room. Procedure-specific trays contained all the components required to insert a cannula. Nine participants managed to gather cannulation equipment in a mean time of $41 \mathrm{~s}$ (range 21-63s). However, 1 (11\%) participant failed to gather all the necessary equipment.

PDSA cycle 3 involved labelling the tray with a crib sheet (listing all components required for the procedure). Thirteen participants gathered the equipment in a mean time of $18 \mathrm{~s}$ (range 7-35s). All participants managed to gather all of the equipment successfully.

In PDSA cycle 4, 13 were timed gathering equipment for cannulation from the customised plastic tray labelled with a crib sheet. All participants gathered all the equipment in a mean time of $18 \mathrm{~s}$ (range 8-35 s), demonstrating a further reduction of $23 \mathrm{~s}$ when compared with PDSA cycle 2 (figure 3 ).

In order to prove our concept works for other procedures, we trialled our procedure-specific tray principal for lumbar puncture and urinary catheter insertion.

This demonstrated the importance of the crib sheets when without this only two out of seven managed to gather the necessary equipment to carry out a lumbar puncture. For the two participants that were successful at completing this task, the mean time taken was $336 \mathrm{~s}$ (range 293-378s).

For the lumbar puncture tray, introduction of the crib sheet decreased the mean time for gathering equipment to $43 \mathrm{~s}$ (range $33-58 \mathrm{~s}$ ). All seven participants managed to gather $100 \%$ of equipment.

We additionally proved this concept by introducing a urinary catheter procedure-specific tray with the crib sheet. Seven participants managed to gather 100\% of equipment in a mean time of 46s (range 34-74s) compared with a mean of 93s (range 66-114s). One participant was unsuccessful at gathering the equipment without the crib sheet. Overall, we demonstrated a mean time saving of $48 \mathrm{~s}$.

\section{LESSONS AND LIMITATIONS}

The first obstacle we encountered was gaining support from ward sisters to incorporate our trays. Issues raised included concerns of whom would be responsible for restocking of equipment and disruption to the treatment room while new trays are being installed. If repeating the project again, we would include nursing staff in our initial survey questionnaire, thus demonstrating that their suggestions were addressed. This may have also highlighted at an earlier stage the issues surrounding infection control. To overcome these issues, we presented at the nursing huddle and met with the infection control team. Once the benefits of the project was demonstrated, support was gained. We contacted the manufacturers to purchase plastic tray dividers, which were in keeping with infection control protocols.

If repeating this QI project, we would start earlier in our clinical rotation so we had more time to collect more data. We would engage with the finance department from the point that we demonstrated the data collection to be statistically significant. This may have prevented delays in ordering the plastic customised trays as the budget would have been clear from the start.

Early contact with the manufacturing company would have provided us with the total cost of roll-out to present to the finance department.

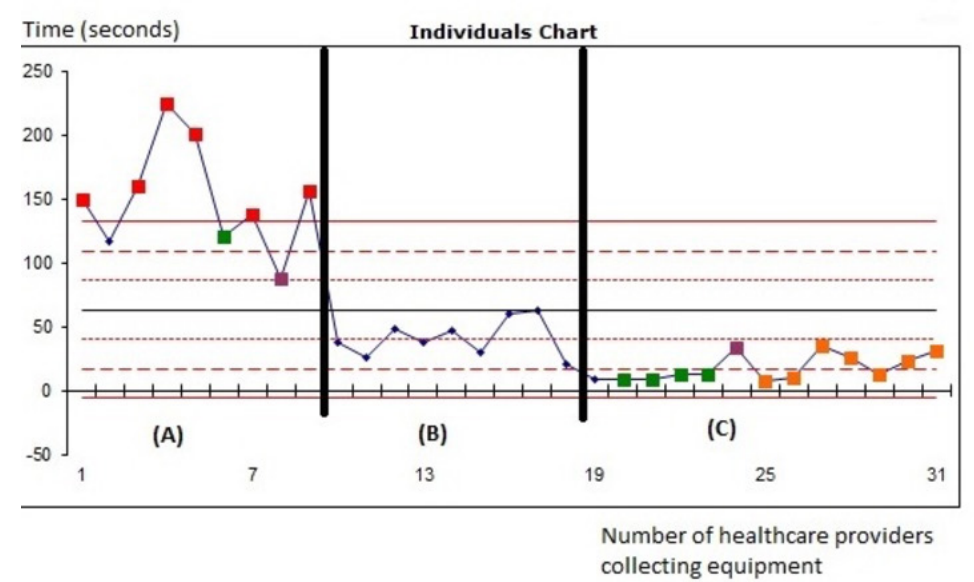

Figure 3 Statistical process chart for gathering equipment for cannulation.
(A) Time taken to gather equipment for cannulation in current layout of treatment room in Acute Medicine ward in NGH.

(B) Time taken to gather equipment for cannulation from labelled drawer containing all needed equipment.

\section{(C) Time taken to gather} equipment for cannulation from labelled drawer containing all needed equipment with the addition of a 'crib sheet'. 
Encouraging collaboration with different stakeholders proved difficult, resulting in delays in progression. In attempting a similar-scale project again, we would arrange regular meetings with all stakeholders present to allow more free discussion of processes and cost of implementation. This may have highlighted the different treatment room sizes and needs. Presenting at the hospital board meeting facilitated the start of these discussions.

We have started preliminary discussions with the manufacturers regarding scale-up and roll-out throughout the hospital. We recognise that, due to time constrains of our 1-year rotation at $\mathrm{NGH}$, for sustainability, we will need to appoint a new junior doctor-led team to oversee the roll-out and implementation. The project will continue to be supported by the hospital QI lead and Patient Safety Board.

To ensure that the project is a success, it is essential that trays are restocked. To achieve this, we have discussed appointing a staff member with the sole responsibility of restocking the trays, which we monitor and adapt during scale-up and roll-out.

\section{CONCLUSION}

Following difficulty in gathering access to medical equipment, we made treatment room codes available on trust-issued iPads and implemented the one-tray procedure-specific concept in a busy acute admissions ward.

Before commencing this project, we compared our ideas with existing projects. We found both accessing and gathering of medical equipment in a timely manner to be a common problem faced by many health professionals within a hospital setting. Our research showed that several previous attempts have been made to organise equipment in the treatment room. These projects have failed due to insufficient handover of the project when rotating to a new hospital each year. We hope to have achieved this effective handover by handing over in person and having the support of the hospital board and QI team give some continuity. From our literature review, no one had attempted procedure-specific tray concept, thus making this a novel endeavour.

Through our various PDSA cycles, we designed compartment dividers and crib sheets in order to improve time efficiency. We surpassed our SMART aim of achieving $25 \%$ efficiency savings for our procedure-specific tray.

In hindsight, we would like to have run PDSA cycles $1-4$ on all of the medical procedures included in this project. We would also have liked to have a larger participant sample size. In order to ensure that this is a lasting and sustainable change, we would like to reaudit this project post roll-out throughout the hospital.

On reflection, we have proven that the procedure-specific trays save time, which junior doctors can use to priorities other tasks. From discussions with junior doctors, they feel more time can be spent delivering patient care.

This project has been well received as we have shown to improve time efficiency, thereby gaining support from the relevant stakeholders. The ongoing support has allowed us to initiate scale-up and roll-out throughout the hospital. If the results obtained are also replicable on a large-scale roll-out throughout $\mathrm{NGH}$, we hope that other hospitals will also follow suit.

Acknowledgements We would like to credit Philip Pearson, Celia Warlow and Jane Bradley of Northampton General Hospital who have mentored us throughout the project. Specifically they have contributed to the methodology and data analysis. They also critically reviewed our article prior to submission.

Contributors YK, AH and HS are all joint authors and have contributed equally in the concept, design, analysis, writing or revision of the manuscript. YK was designated as corresponding author.

Competing interests None declared.

Provenance and peer review Not commissioned; externally peer reviewed. Open Access This is an Open Access article distributed in accordance with the Creative Commons Attribution Non Commercial (CC BY-NC 4.0) license, which permits others to distribute, remix, adapt, build upon this work non-commercially, and license their derivative works on different terms, provided the original work is properly cited and the use is non-commercial. See: http://creativecommons.org/ licenses/by-nc/4.0/

(C) Published by the BMJ Publishing Group Limited. For permission to use (where not already granted under a licence) please go to http://www.bmj.com/company/ products-services/rights-and-licensing/

\section{REFERENCES}

1. Pedley R, Whitehouse A, Hammond S. Improving room layouts for venepuncture, cannulation and $A B G$ equipment on surgical wards. BMJ Qual Improv Rep 2014;2:u554.w477.

2. Lindley S, Robertson I. A standardised storage solution for venepuncture/cannulation equipment could save an NHS hospital the equivalent of a whole junior doctor. BMJ Qual Improv Rep 2014;2:u202220.w1207.

3. Ward J, Spencer R, Soo E, et al. Standardising the organisation of clinical equipment on surgical wards at North Bristol NHS Trust: a quality improvement initiative. BMJ Qual Improv Rep 2015;4:u208308. w3441.

4. Peng M, Lovett S, Damda F. G216(P) The treatment room on children's ward - a quality improvement project. Arch Dis Child 2014;99:A93.

5. Clements A, McFarquhar A, Bhatti A. Improving venepuncture in district general hospital. Quality Improvement Project at Northampton General Hospital. 2015. 